\title{
Dispersión de larvas de crustáceos decápodos en canales y fiordos de la Región de Aysén, Chile
}

\author{
Dispersion of decapods crustacean larvae in channels and fjords of the Aysen Region, Chile
}

\author{
Armando Mujica R. \\ Departamento de Acuicultura, Facultad de Ciencias del Mar, Universidad Católica del Norte \\ Sede Coquimbo. Casilla 117, Coquimbo, Chile \\ amujica@ucn.cl
}

\begin{abstract}
Distribution and abundance of planktonic subadult forms of decapods crustaceans was studied in the channels and fjords of the Aysen region in austral Chile (43$46^{\circ} \mathrm{S}$ ) during November 2003. Larval dispersal of the most abundant species (Sergestes arcticus, Neotrypaea uncinata and Munida subrugosa) is discussed.

Caridea, Thalassinidea and Brachyura were the most abundant infraorders. Thalassinidea was represented by a single species (Neotrypaea uncinata), the most abundant in this study and one of the three that due to its ample distribution allowed us to offer an explanation for its dispersion based on the larval stages composition of samples at each sampling station.

Neotrypaea uncinata and Munida subrugosa larvae exhibited a more oceanic distribution than the larvae of Sergestes articus whose cosmopolitan adults display benthopelagic distribution that could explain its presence in the inner channels.
\end{abstract}

Key words: Meroplankton, distribution, Austral channels
Resumen.- Se estudió la distribución y abundancia de larvas de crustáceos decápodos en los canales y fiordos de la

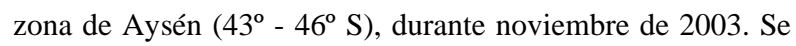
discute el posible desplazamiento de las larvas de las especies más abundantes (Sergestes arcticus, Neotrypaea uncinata y Munida subrugosa).

Los infra órdenes más abundantes fueron Caridea, Thalassinidea y Brachyura. Thalassinidea estuvo representado por una sola especie (Neotrypaea uncinata), que fue la de mayor abundancia relativa del estudio y una de las tres que por su amplia distribución ha permitido dar una interpretación de su dispersión, basada en la composición por estados de desarrollo en las muestras de cada estación de muestreo.

Las larvas de Neotrypaea uncinata y Munida subrugosa presentaron una distribución más oceánica que las de Sergestes arcticus, cuyos adultos cosmopolitas son de hábitos bentopelágicos, lo que podría ser la causa de su presencia en canales interiores.

Palabras clave: Meroplancton, distribución, canales australes

\section{Introducción}

La información generada en los cruceros oceanográficos Cimar Fiordo I, IV y VIII, que prospectaron la zona

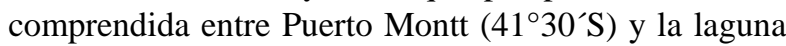
San Rafael ( $\left.46^{\circ} 40^{\prime} \mathrm{S}\right)$, Chile, durante la primavera de 1995 , la primavera y verano de 1998-99 y la primavera de 2002, respectivamente, permitió que la comunidad científica nacional a través del Comité Oceanográfico Nacional, haya divulgado importante información oceanográfica de canales y fiordos de la Región de Aysén.

La variedad ambiental de los canales, fiordos y senos de esta región es una de las causas de la riqueza de especies de crustáceos decápodos que en ellos se encuentra (Henríquez \& Bahamonde 1976, Retamal 1981, 2000). Las condiciones físicas, químicas y biológicas de la columna de agua y circulación e intercambio de aguas interiores y oceánicas de la región, constituyen una zona de reproducción, crianza y dispersión de poblaciones y recursos pelágicos y bentónicos marinos poco estudiada.

La información zooplanctónica emanada de los cruceros Cimar Fiordos constituye los primeros antecedentes integrados de esta área del conocimiento para la región, los que relacionan aspectos oceanográficos con el ictioplancton (Balbontín \& Bernal 1997), carcinoplancton (Mujica \& Medina 1997, Mujica 2002, 2003) y zooplancton gelatinoso (Palma \& Rosales 1997, Palma \& Aravena 2002). 
Como consecuencia de la información generada en esta área del conocimiento, el Comité Oceanográfico Nacional (CONA), en el desarrollo del crucero Cimar Fiordo IX contempló entre otras, la investigación oceanográfica relacionada con la deriva y retención larval de organismos nectónicos y bentónicos y su relación con variables físicas y químicas de los ambientes estuarinos que constituyen los diferentes y dinámicos canales y fiordos de la zona de Aysén (CONA 2004).

El presente trabajo entrega nuevos antecedentes de la composición, abundancia y dispersión de las larvas de crustáceos decápodos de los principales canales y fiordos de la región de Aysén, como complemento de la información oceanográfica generada en los programas de investigación del Comité Oceanográfico Nacional.

\section{Material y métodos}

En 31 estaciones oceanográficas ubicadas en la zona de los canales de la Región de Aysén, comprendidos entre la Boca del Guafo y Bahía Ana Pink (Fig. 1), se tomaron muestras zooplanctónicas entre el 7 y 11 de noviembre de 2003, en forma continua (día y noche).



Figura 1

Mapa con la ubicación de estaciones oceanográficas

Map with location of oceanographic stations 
Las muestras fueron obtenidas a bordo del $\mathrm{B} / \mathrm{O}$ AGOR Vidal Gormaz, mediante el uso de redes bongo de $59 \mathrm{~cm}$ de diámetro de boca y $300 \mu \mathrm{m}$ de abertura de malla, provistas de medidores de flujo. Ellas fueron arrastradas en forma doble oblicua entre la superficie y $200 \mathrm{~m}$ de profundidad. Las muestras obtenidas fueron fijadas en solución neutralizada de formalina en agua de mar al 5\%, para su posterior análisis en laboratorio. De cada muestra se extrajo la totalidad de larvas de decápodos, que fueron contadas e identificadas a nivel de familia, género, especie, dependiendo de la información existente de los estadios de desarrollo de ellas. Las larvas de las especies más abundantes (Sergestes arcticus, Neotrypaea uncinata y Munida subrugosa) fueron separadas por estadios de desarrollo.

El número de ejemplares capturados fue estandarizado como número de larvas presentes en $1000 \mathrm{~m}^{3}$ de agua filtrada, información que permitió determinar la abundancia, la dominancia numérica (porcentaje de cada grupo, respecto del total capturado), frecuencia de ocurrencia (porcentaje de estaciones con larvas, respecto del total de estaciones efectuadas).

\section{Resultados}

Se analizaron 12.875 larvas y juveniles de crustáceos decápodos, identificados como componentes de los subórdenes Dendobranchiata y Pleocyemata. Del primero de ellos, sólo se identificaron larvas de Sergestes arcticus en estados de desarrollo elaphocaris, acanthosoma, mastigopus y juveniles, predominando los primeros. Las larvas de esta especie aunque tuvieron baja dominancia numérica $(5,6 \%)$ presentaron amplia distribución (Tabla 1), predominando en los canales interiores y la parte sudoccidental de la zona de muestreo (Fig. 2). En general, los estadios elaphocaris fueron más abundantes en las estaciones de los canales interiores, mientras que los estadios acanthosoma, se distribuyeron principalmente en las estaciones oceánicas de la parte sur. Los estadios mastigopus y juveniles se encontraron en pequeñas concentraciones en estaciones del canal Moraleda y próximas a él. No se encontraron larvas de esta especie en estaciones de los canales Darwin y Pulluche. La mayor abundancia, con valores próximos a 300 larvas $1.000 \mathrm{~m}^{-3}$, se encontró en la entrada del seno Aysén y canal Puyuguapi (Fig. 2).

Tabla 1

Abundancia relativa, dominancia numérica y frecuencia de ocurrencia de larvas de crustáceos decápodos (Cimar 9, Fiordos Región de Aysén)

Relative abundance, numerical dominance and frequency occurrence of decapod crustacean larvae (Cimar 9, Fiordos, Aysen Region)

\begin{tabular}{lccc}
\hline Orden Decapoda & $\begin{array}{c}\text { Abundancia } \\
\left(\mathrm{N}^{\mathrm{o}} 1000 \mathrm{~m}^{-3}\right)\end{array}$ & $\begin{array}{c}\text { Dominancia } \\
\text { numérica (\%) }\end{array}$ & $\begin{array}{c}\text { Frecuencia de } \\
\text { ocurrencia (\%) }\end{array}$ \\
\hline $\begin{array}{l}\text { Subord. Dendrobranchiata } \\
\text { Superfam. Sergestoidea }\end{array}$ & 1.393 & 5,6 & 74,2 \\
$\begin{array}{l}\text { Subord. Pleocyemata } \\
\text { Infraord. Caridea }\end{array}$ & 5.646 & 22,7 & 100 \\
Infraord. Thalassinidea & & & \\
Fam. Callianassidae & 2.365 & 9,5 & 77,4 \\
Infraord. Anomura & & & \\
Fam. Albuneidae & 1 & $<0,1$ & 3,2 \\
Fam. Lithodidae & 2 & $<0,1$ & 3,2 \\
Fam. Paguridae & 177 & 0,7 & 80,6 \\
Fam. Galatheidae & 1.334 & 5,4 & 64,5 \\
Fam. Porcellanidae & 662 & 2,7 & 100 \\
Infraord. Brachyura & 11.389 & 45,8 & \\
\hline
\end{tabular}




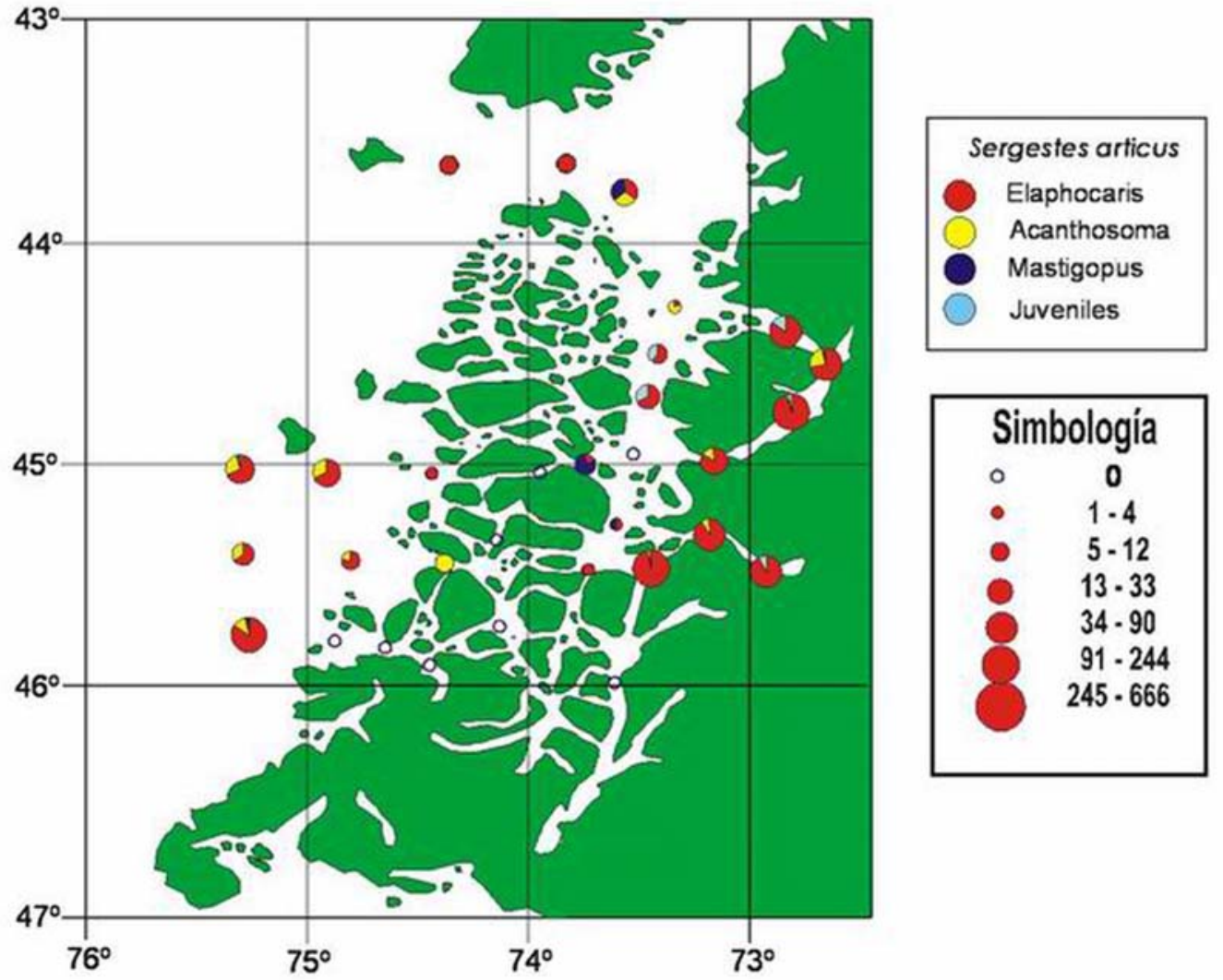

Figura 2

Distribución y abundancia de larvas de Sergestes arcticus

Distribution and abundance of Sergestes arcticus larvae

Del suborden Pleocyemata, se capturaron larvas y juveniles de los infraórdenes, Caridea, Thalassinidea, Anomura y Brachyura (Tabla 1).

Las larvas de Caridea se encontraron en todas las estaciones muestreadas. Fue el segundo infraorden en abundancia y representaron más del 22\% del total de larvas capturadas (Tabla 1). Las máximas concentraciones de estas larvas (700 a 1.279 larvas $1.000 \mathrm{~m}^{-3}$ ), se encontraron en estaciones ubicadas en la parte occidental del canal Ninualac.

Entre las larvas más desarrolladas de este infraorden, se pudo identificar ejemplares de Pasiphaea sp., Campylonotus sp., Austropandalus grayi y Nauticaris magellanica.
Del infraorden Thalassinidea sólo se encontraron larvas y juveniles de Neotrypaea uncinata, especie perteneciente a la familia Callianassidae. Las larvas de $N$. uncinata fueron las más abundantes, dominantes y frecuentes de las especie identificada en el estudio (Tabla 1). Predominaron los estadios zoeas, encontrándose megalopas y juveniles en pequeñas concentraciones sólo en la estación 65, ubicada en la entrada occidental del canal Darwin. Las máximas concentraciones (658 larvas $1.000 \mathrm{~m}^{-3}$ ) se detectaron en esta estación y en las estaciones 5 y 63, ubicadas en la entrada del canal Moraleda y Ninualac, (220 y 282 larvas $1.000 \mathrm{~m}^{-3}$, respectivamente). En las estaciones ubicadas en los canales orientales no se detectó su presencia (Fig. 3). 


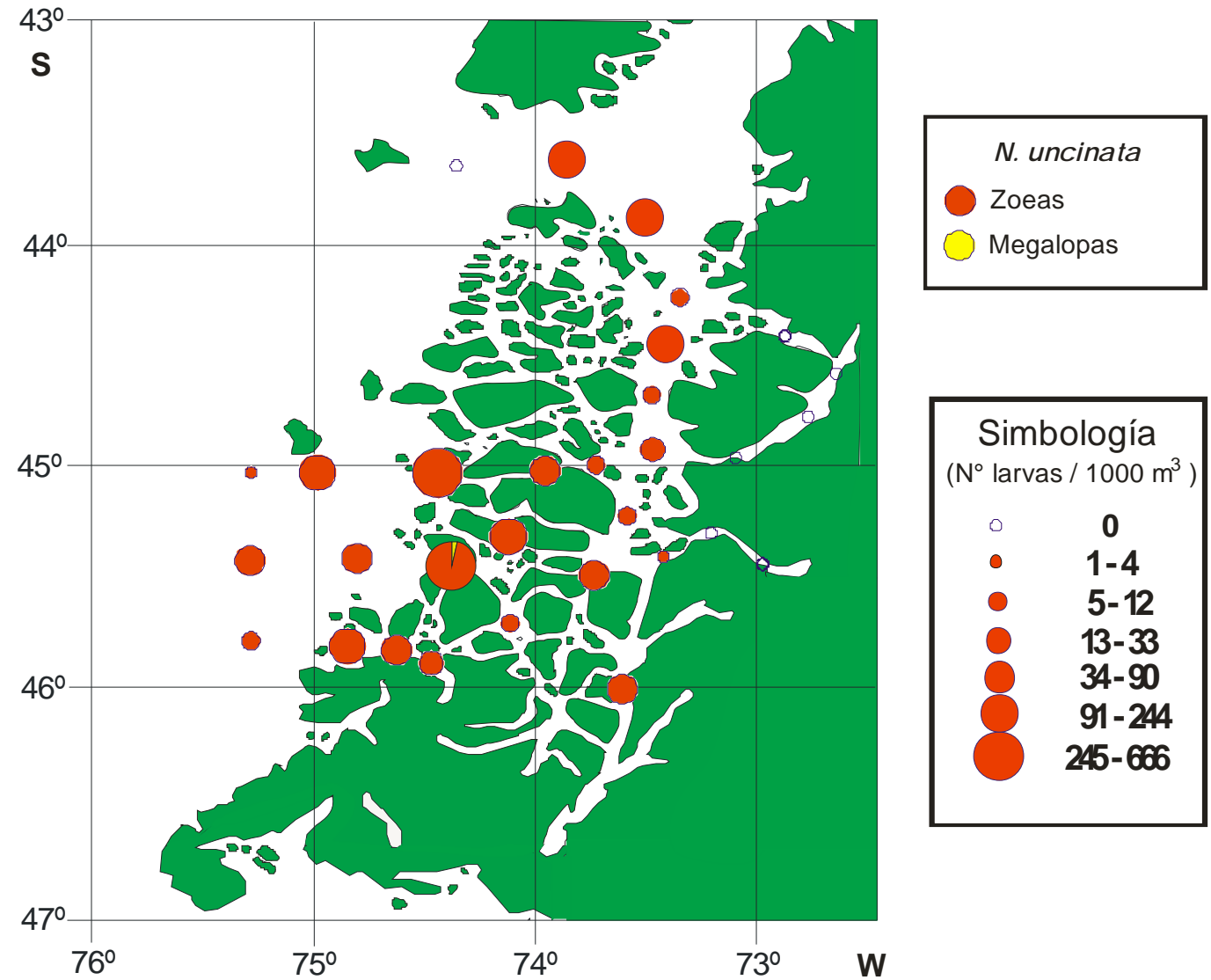

Figura 3

Distribución y abundancia de larvas de Neotrypaea uncinata

Distribution and abundance of Neotrypaea uncinata larvae

Del infraorden Anomura se identificaron larvas de cinco familias (Tabla 1). De la familia Albuneidae, se encontraron larvas zoeas de Blepharipoda spinimana, en mínimas concentraciones en una sola estación, ubicada en la boca del Guafo (Fig. 4).

De igual forma, las larvas de Lithodidae fueron capturadas en mínimas concentraciones, sólo en una estación ubicada en la entrada occidental del canal Ninualac (Fig. 4). Ellas fueron identificadas como estadios megalopas de Lithodes santolla.

De la familia Paguridae se capturaron larvas en el $58,1 \%$ de las estaciones, aunque en muy bajas concentraciones (Tabla 1). Su abundancia máxima fue de 30 larvas $1.000 \mathrm{~m}^{-3}$ y predominaron las zoeas por sobre las megalopas, las cuales sólo se capturaron en tres estaciones: una ubicada en el canal Moraleda y las dos restantes en la boca del Guafo y próxima a la isla Guamblin, ambas en el sector occidental del área de estudio, que es el de mayor influencia oceánica (Fig. 4).

De la familia Galatheidae se capturaron larvas de Munida subrugosa en el 80,6\% de las estaciones, lo que las constituyó en las larvas de distribución más amplia en la zona de estudio. Su abundancia relativa fue similar a la de Sergestes arcticus, que en conjunto con las larvas de Neotrypaea uncinata, las constituyó en las más abundantes del estudio (Tabla 1).

Las mayores concentraciones de larvas de $M$. subrugosa se hallaron en el sector sudoccidental de la zona de muestreo, con concentraciones comprendidas entre 100 y 300 larvas $1.000 \mathrm{~m}^{-3}$, mientras que en las 


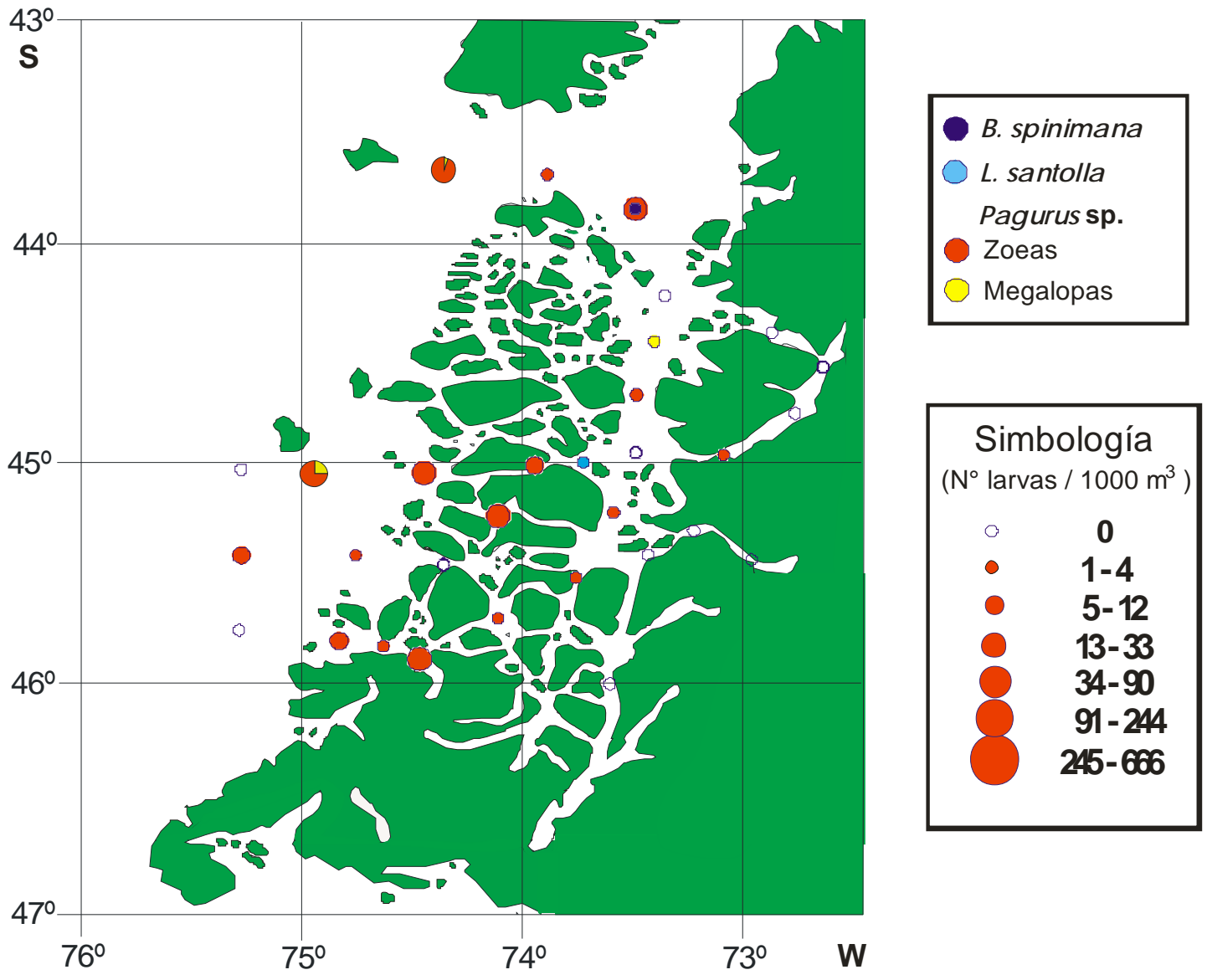

Figura 4

Distribución y abundancia de larvas de Blepharipoda spinimana, Lithodes santolla y Pagurus sp.

Distribution and abundance of Blepharipoda spinimana, Lithodes santolla and Pagurus sp. larvae

estaciones de la parte oriental (canal Moraleda, Puyuguapi y seno Aysén), fueron menos abundantes o estuvieron ausentes (Fig. 5). El 84,6\% de estas larvas corresponden a estados de zoeas, de las cuales el 54,6\% fueron de los primeros estados de desarrollo (zoeas sin urópodos), las que predominaron en las estaciones ubicadas desde el canal Moraleda al poniente. Las zoeas más desarrolladas (con urópodos), se hallaron también en estas estaciones y en los canales de la parte oriental de la zona de estudio (Fig. 5). Las larvas megalopas, que sólo constituyeron el 15,4\% de la especie, se capturaron principalmente en estaciones de la parte sudoccidental (isla Guamblin, canales Ninualac, Darwin y Puluche (Fig. 5).
Las larvas de la familia Porcellanidae correspondieron a zoeas de dos morfotipos del género Petrolisthes, que difieren entre sí por la longitud de las espinas del caparazón y la ornamentación de las setas del telson. Se capturaron en el 64,5\% de las estaciones y su dominancia numérica sólo fue de $2,7 \%$ (Tabla 1). Se encontraron principalmente en los canales del sector occidental y sus máximas abundancias fueron registradas en la entrada occidental del canal Ninualac (326 larvas $1.000 \mathrm{~m}^{-3}$ ), única localidad en la que se capturó un ejemplar en estado de megalopa (Fig. 6). 


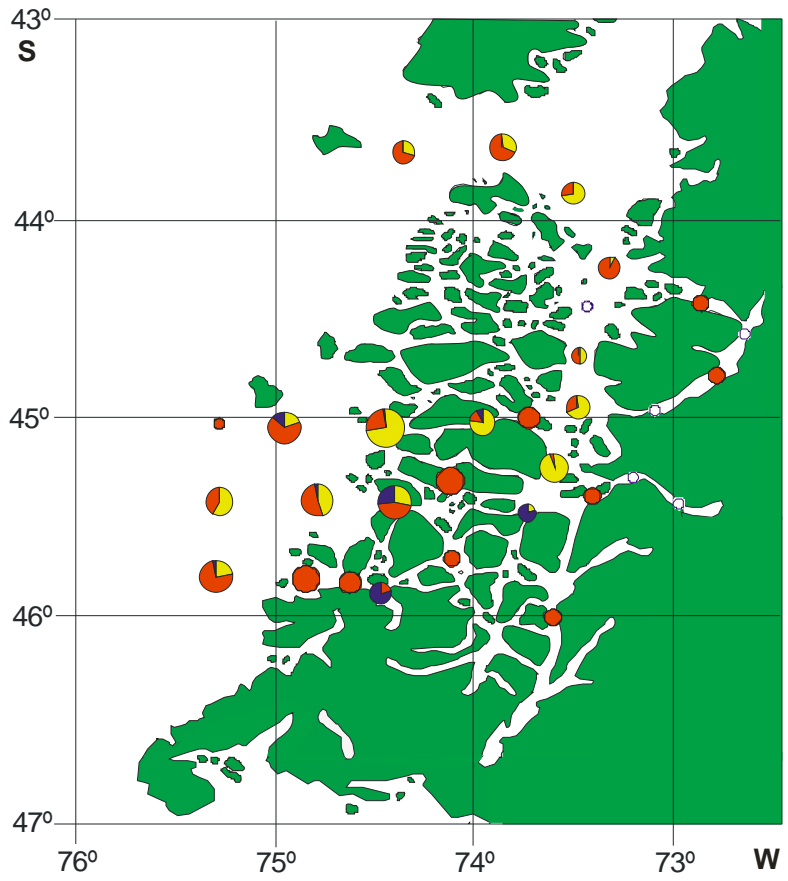

Munida subrugosa

Zoea sin uropodos

Zoea con uropodos

Megalopa

Figura 5

Distribución y abundancia de larvas de Munida subrugosa

Distribution and abundance of Munida subrugosa larvae

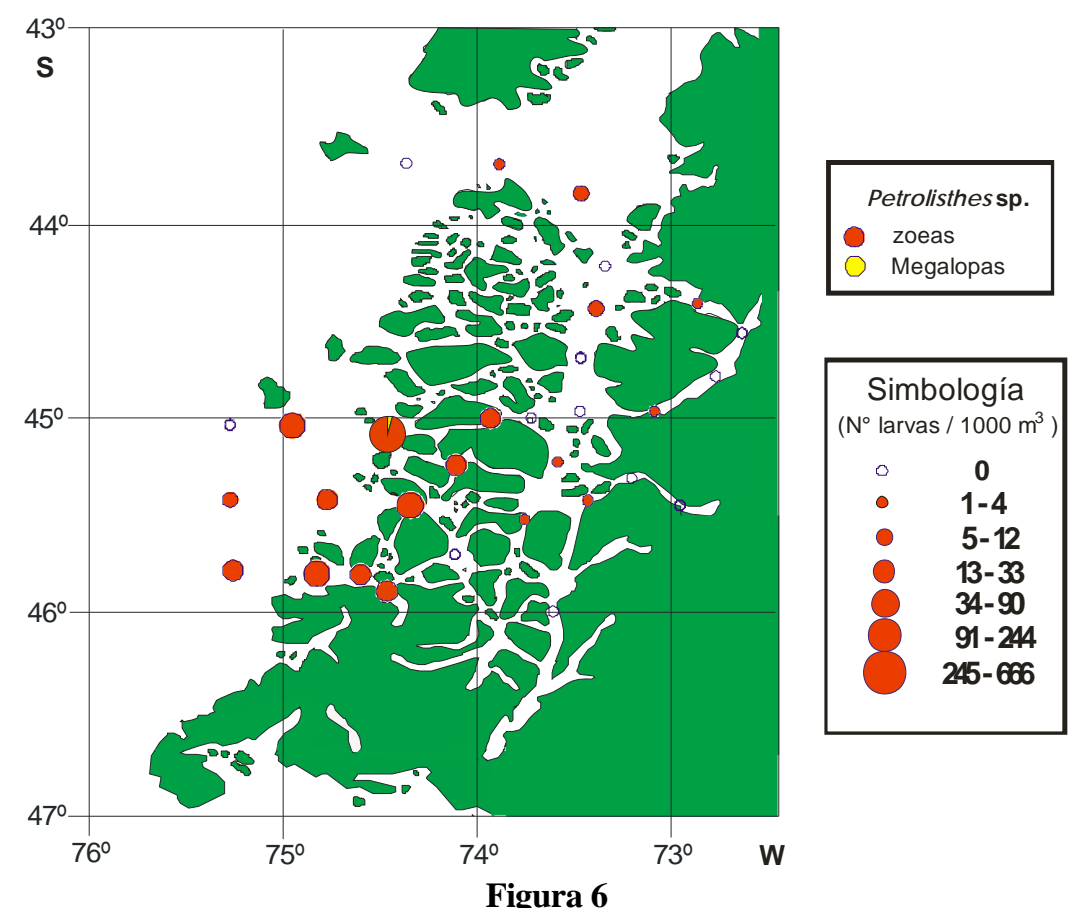

Distribución y abundancia de larvas de Petrolisthes sp.

Distribution and abundance of Petrolisthes sp. larvae 
Larvas del infraorden Brachyura se encontraron en la totalidad de las estaciones muestreadas, predominando los primeros estados de desarrollo (zoeas). Como grupo fueron las más abundantes del estudio, con dominancia de 45,8\%. La mayores concentraciones se encontraron en las estaciones del sector sudoccidental del área de estudio, con máximos de 1.828 y 2.323 larvas $1.000 \mathrm{~m}^{-3}$, en las estaciones 63 y 67, respectivamente. Entre los ejemplares identificados, que corresponden sólo a parte de este infraorden, se encontraron larvas de Eurypodius latreillei, Pisoides edwardsi, Halicarcinus planatus, Corystoides chilensis, dos morfotipos del género Cancer y dos del género Pinnixia. Los ejemplares de este último género fueron los de mayor abundancia y amplia distribución, seguidos de las larvas de Cancer.

\section{Discusión}

En general, la abundancia relativa de las larvas de decápodos fue inferior a la registrada en similar área de estudio en años anteriores (Mujica \& Medina 1997, Mujica 2002, 2003). Los trabajos antes señalados, tomaron muestras la zona al comienzo de primavera (septiembre - octubre), época que coincide con la mayor concentración de larvas de crustáceos decápodos en el plancton, lo que explicaría la mayor abundancia respecto de esta oportunidad, en la que el muestreo se efectuó en noviembre.

Mujica (2003) compara la abundancia y composición de estado de desarrollo de las larvas de crustáceos decápodos capturadas en la zona de Aysén, entre septiembre y octubre de 1998 y febrero de 1999, indicando la notoria disminución de la abundancia de las larvas de la mayoría de las especies identificadas en el segundo crucero, así como el aumento proporcional de los estados de desarrollo más avanzados.

De las especies identificada, las larvas de Sergestes arcticus fueron una de las tres especies más abundantes y de amplia distribución, lo que coinciden con lo señalado para ellas en la zona de muestreo (Mujica \& Medina 1997, Mujica 2002, 2003). S. arcticus es un camarón de hábitos bentopelágicos de muy amplia distribución, que ha sido descrito para las costas de Chile desde Valparaíso a Punta Arenas y el archipiélago de Juan Fernández (Retamal 1981, 2000). También se ha descrito su presencia en aguas del Atlántico Norte, mar Mediterráneo, islas Canarias, Sud África, costas de Uruguay, Australia y Nueva Zelanda
(Boschi 1981, Retamal 1981, Retamal 2000), lo que la constituye en una especie cosmopolita.

Las larvas encontradas en esta oportunidad, coinciden con la descripción de Boschi (1981) y Mujica (2002, 2003), para los diferentes estados de desarrollo. Su amplia distribución en este estudio, coincide con los resultados encontrados por Mujica \& Medina (1997) y Mujica (2002, 2003), así como las zonas de mayores concentraciones, centrada en el seno Aysén y canal Puyuguapi, fiordos en los que el recambio de aguas es menos dinámico, además de confluir aguas continentales y oceánicas (Silva et al. 1997). La variabilidad transversal del flujo de las aguas de los canales de la región que describe Cáceres et al. (2004), indican la complejidad de su dinámica. Estos autores, indican que esta dinámica es producto de la interacción del efecto del viento, la topografía submarina y las corrientes de marea. Al respecto se puede señalar, que la dispersión de las larvas planctónicas que no logran la capacidad natatoria para vencer las corrientes, estaría determinada por el efecto de estas corrientes, no obstante que la migración vertical diaria que ellas efectúan, podría ser uno de los mecanismos que les permite retornar o permanecer en áreas oceanográficas favorables para su desarrollo (Chen et al. 1997, Martinho et al. 2006). Al respecto, Balbontín et al. (2004), analizan la distribución vertical del meroplancton, señalando que las larvas de $S$. arcticus no siguen el patrón de migración vertical circadiario.

El predominio de los primeros estados de desarrollo de $S$. arcticus en toda la zona muestreada, indican la amplia distribución de los adultos, mientras que la distribución de larvas mastigopus y juveniles, centrada en las estaciones del canal Moraleda e inmediaciones, indicaría que las larvas elaphocaris que abundan en los canales interiores serían transportadas hacia el canal Moraleda. Al respecto, Mujica (2002), al comparar las capturas de dos épocas sucesivas en el sector, encontró mayor abundancia de los primeros estadios en los canales interiores y sólo mastigopus y juveniles en la entrada de los canales y zonas de mayor influencia oceánica. Por tanto, descarta la posibilidad de diferencias geográficas en la época reproductiva de la especie. En consecuencia, la distribución segregada de los estados de desarrollo de la especie indicaría el transporte de las larvas durante el transcurso de su desarrollo, desde la parte interior de los canales hacia el canal Moraleda, posiblemente encontrando en éste, las características oceanográficas adecuadas para que se verifique el reclutamiento de la especie. 
La gran abundancia y frecuencia de ocurrencia de las larvas de Caridea y Brachyura, es producto de la riqueza específica de los adultos que han sido descritos para la zona de estudio (Retamal 1981, 2000). Mujica \& Medina (1997, 2000) y Mujica \& Villablanca (2003), coinciden en señalar que la amplia distribución y gran abundancia relativa de estas larvas, corresponden con la gran variedad de especies de los infraórdenes. Retamal (1981, 2000) describe más de diez especies de Caridea, que pertenecen a siete familias (Pasiphaeidae, Oplophoridae, Pandalidae, Alpheidae, Hippolytidae, Campylonotidae y Crangonidae), que en su distribución geográfica involucrarían la zona de estudio. Respecto de Brachyura, el 41\% de las especies de crustáceos decápodos citados para los canales australes, corresponden a este infraorden (Retamal 2000). Al respecto y dado que la identificación de las larvas de Caridea y Brachyura ha sido parcial, de su presencia, abundancia y distribución, no se pudo deducir algún patrón de dispersión de ellas.

La distribución de las larvas de Neotrypaea uncinata coincide con lo señalado por Mujica \& Medina (1997) y Mujica (2002, 2003) para la zona de muestreo, quienes encontraron las mayores abundancias en los canales poco profundos de influencia oceánica y ausentes en su interior. La distribución batimétrica de los adultos de esta especie ha sido señalada para rangos comprendidos entre 0 y $1 \mathrm{~m}$ (Retamal 1981, 2000), que coincide con la captura de las mayores concentraciones de sus larvas en estaciones de poca profundidad, las cuales serían arrastradas por corrientes hacia lugares de mayor profundidad, en los que se capturaron en menores concentraciones. La presencia de estadios avanzados de desarrollo (megalopas) en zonas protegidas de poca profundidad, en correspondencia con los hallazgos de Mujica (2003) en similar zona de estudio, sugiere alguna estrategia de retención que tendrían las larvas para retornar o permanecer en lugares en los que encontrarán condiciones propicias para su vida bentónica adulta. Al respecto, se ha señalado que la migración vertical circadiaria que las larvas de crustáceos realizan como parte del plancton y la migración ontogénica de los decápodos con larvas pelágicas, participan directamente en el proceso de retención y dispersión larval, permitiendo que la descendencia de la carcinofauna bentónica o pelágica, encuentre un ambiente propicio para completar su desarrollo (Chen et al. 1997, Escribano et al. 2002, Martinho et al. 2006, Shanks 2006).
Las larvas de Blepharipoda spinimana encontradas en esta oportunidad coinciden con lo reportado por Mujica \& Medina (1997), Mujica (2002, 2003) para esta zona de muestreo. Estos autores discuten su presencia respecto de la distribución de los adultos, señalando que ellas se encuentran al sur de su rango de distribución. Los adultos de esta especie han sido descritos desde Arica a Mehuin (Retamal 2000), por lo que su presencia en el plancton en la localidad en que fueron capturadas y el incipiente estado de desarrollo de ellas, se debería a la presencia de adultos en los canales y playas próximas a los lugares de captura de estas larvas.

Las larvas de Galatheidae corresponden con las identificadas por Mujica (2002), quien discute los argumentos disponibles y la morfología larval, señalando que pertenecen a Munida subrugosa. Los adultos de esta especie son de hábitos bentopelágicos y formas importantes agregaciones en las costas atlántica y pacífica del extremo sur de América del Sur (Matthews 1932, Rayner 1935, Retamal 1981). Su amplia distribución y frecuencia de ocurrencia en las muestras planctónicas de los fiordos australes, ha sido anteriormente descrito (Mujica \& Medina 1979, 2000, Mujica 2002, 2003, Mujica \& Villablanca 2003). Las larvas de $M$. subrugosa que estos autores capturaron en los fiordos y canales comprendidos entre el seno Reloncaví y el Cabo de Hornos, fueron más abundantes en estaciones ubicadas en canales con importante aporte de aguas oceánicas, característica que se mantuvo en este estudio. Mujica (2003), al comparar la composición por estado de desarrollo de estas larvas en los canales de la zona de Aysén en épocas sucesivas, comprobó el aumento progresivo de la proporción de las larvas de desarrollo más avanzado en verano, respecto de primavera. En esta oportunidad, en que el muestreo fue efectuado a mediado de la primavera, la abundancia de los estados de zoeas avanzados, fueron similares a las abundancias de las zoeas de desarrollo temprano, mientras que las megalopas aunque en bajas concentración tuvieron amplia distribución. La distribución de los adultos de esta especie, por tener hábitos bentopelágico al igual que $S$. articus, no depende de las características del sustrato como es el caso de $N$. uncinata, que es una especie propia del bentos intermareal somero blando, por lo que sus larvas están estrechamente asociadas a zonas de estas características, desde la cual se dispersan. La amplia distribución y frecuencia de ocurrencia de las larvas y adultos de $M$. subrugosa se debería al rango de tolerancia que tienen para vivir en las variadas 
condiciones ambientales de los canales, lo que permitiría que la actividad reproductiva de la especie se verifique en amplias zonas.

La composición por estadios de desarrollo de $M$. subrugosa y en particular la distribución de las megalopas, que coincide con los estadios más tempranos de desarrollo, permite suponer que la dispersión o retención entre ellas es similar, puesto que de larva a adulto, esta especie sólo cambiaría su capacidad natatoria, lo que dada las velocidades de flujo descrito para canales de la zona de estudio (Cáceres 2004), los adultos también serían arrastrados por las corrientes de los canales.

Balbontín et al. (2004) encuentran que la distribución vertical de estas larvas es parcialmente opuesta a la migración vertical diaria del zooplancton en general, lo cual podría ser explicado por la dinámica de los canales, cuyas corrientes de marea superarían las capacidades de desplazamiento vertical de las larvas.

\section{Agradecimientos}

El autor agradece al Comité Oceanográfico Nacional que permitió mediante el financiamiento y gestión, la toma de muestras y ejecución a este trabajo. A la Facultad de Ciencias del Mar de la Universidad Católica del Norte, que puso a disposición del suscrito la infraestructura necesaria para el análisis de la información. Al equipo de investigadores y tripulación del buque AGOR Vidal Gormaz, que colaboraron en la toma de muestras.

\section{Literatura citada}

Boschi E. 1981. Larvas de Crustacea Decapoda. En: Boltovskoy D (ed). Atlas del zooplancton del Atlántico sudoccidental y método de trabajo con el zooplancton marino, pp. 699 - 758. INIDEP, Mar del Plata.

Balbontín F \& B Campos, A Mujica, G Córdova, M Benelli \& C Ugalde. 2004. Distribución vertical de larvas de moluscos, crustáceos y huevos y larvas de peces, en el fiordo Aysén y canal Errázuriz, zona austral de Chile. En: CONA (ed). Crucero Cimar 9 Fiordos. Informes Preliminares, pp. 77-85. Comité Oceanográfico Nacional, Valparaíso.

Cáceres M, A Valle-Levinson, J Fierro, M Bello \& M Castillo. 2004. Variabilidad transversal del flujo submareal en fiordos del mar interior de Chile. En:
CONA (ed). Crucero Cimar 9 Fiordos. Informes Preliminares, pp. 25-35. Comité Oceanográfico Nacional, Valparaíso

Chen Y-H, P-T Shaw \& T G Wolcott. 1997. Enhancing estuarine retention of planktonic larvae by tidal currents. Estuarine, Costal and Shelf Science 45: 525-533.

CONA. 2004. Crucero Cimar 9 Fiordos. Informes preliminares, 151 pp. Comité Oceanográfico Nacional, Valparaíso.

Escribano R, V Marín, P Hidalgo \& G Olivares. 2002. Physical-biological interactions in the pelagic ecosystem of the nearshore zone of the Northern Humboldt Current System. En: Castilla JC \& J Largier (eds). Proceedings of the International Symposium on Linkages and Dynamics of Costal Systems, pp. 145-175. Ediciones Universidad Católica de Chile, Santiago.

Martinho M-A, J Dubert, A Peliz \& H Quetroga. 2006. Influence of vertical migration pattern on retention of crab larvae in a seasonal upwelling system. Marine Ecology Progress Series 307: 1-19.

Matthews L. 1932. Lobster-krill, anomuran Crustacea that are the food of whales. Discovery Reports 5: 467-487.

Mujica A. 2002. Larvas de crustáceos decápodos de los


Doctoral, Departamento de Biología, Universidad de Barcelona, Barcelona, 275 pp.

Mujica A. 2003. Larvas de crustáceos decápodos de la zona de Aysén. Ciencia y Tecnología del Mar 26(2): 109-121.

Mujica A \& M Medina. 1997. Larvas de crustáceos decápodos de los canales australes de Chile (4130' 46³0’ S). Ciencia y Tecnología del Mar 20: 147-154.

Mujica A \& M Medina. 2000. Distribución y abundancia de larvas de crustáceos decápodos en el zooplancton de los canales australes. Proyecto Cimar-Fiordo 2. Ciencia y Tecnología del Mar 23: 49-68.

Mujica A \& R Villablanca. 2003. Larvas de crustáceos decápodos de los fiordos y canales magallánicos. Ciencia y Tecnología del Mar 26 (1): 73-84.

Rayner G. 1935. The Falkland species of the Crustacean Genus Munida. Discovery Reports 10: 209-245.

Retamal M. 1981. Catálogo ilustrado de los crustáceos decápodos de Chile. Gayana Zoología 44: 1-110.

Retamal M. 2000. Decápodos de Chile. 1995. World Biodiversity Database CD-ROM Series. ETI - U. de Concepción. Springer-Verlag, Amsterdam.

Rodríguez O \& $\mathbf{R}$ Bahamonde. 1986. Contribución al conocimiento de Munida subrugosa (White, 1847) en la XII Región, Chile. En: Arana P (ed). La pesca en Chile, pp. 283-296. Ediciones Universitarias, Valparaíso. 